\title{
Steroid induced osteoporosis: an opportunity for prevention?
}

\author{
I David Peat, Susan Healy, David M Reid, Stuart H Ralston
}

\begin{abstract}
Objective-To determine the frequency with which osteoporosis prophylaxis is given to corticosteroid treated hospital inpatients.
\end{abstract}

Methods-All patients receiving systemic corticosteroids in a large teaching hospital over a three month period were identified through routine prescription monitoring by hospital ward pharmacists. Coprescription of antiosteoporotic therapy was recorded, along with other relevant details such as steroid dose, actual, or intended duration of therapy, and indication for therapy.

Results-Corticosteroids were prescribed to 214 patients over the study period, giving an average rate of 2.5 new prescriptions each day. Indications included: chest disease $(n=84 ; 39.2 \%)$, cancer $(n=17 ; 7.9 \%)$, inflammatory bowel disease $(n=16 ; 7 \cdot 5 \%)$ rheumatoid arthritis/connective tissue disease $(n=16$; $7 \cdot 5 \%$, and renal diseases $(n=7 ; 3 \cdot 3 \%)$. One hundred and twelve patients $(52 \cdot 3 \%)$ were receiving short term steroid therapy (less than four months); 66 (37\%) were receiving medium/long term steroid therapy (four months or more). In 36 cases $(16 \cdot 8 \%)$ the duration of therapy was unknown. Only 12 of the 214 patients $(5 \cdot 6 \%)$ received any form of osteoporosis prophylaxis. The prevalence of prophylaxis was similarly low in postmenopausal women (six of $93 ; 6.4 \%$ ) and in patients receiving high dose long term steroid therapy (two of $25 ; 8 \%$ ).

Conclusions-Systemic corticosteroids are used frequently in hospital practice for a wide range of indications, but few patients receive co-prescription of prophylaxis against osteoporosis. This is true even in high risk groups such as postmenopausal women and those on high dose long term steroid therapy. Identification of individuals by the mechanism used in this study provides an opportunity by which all corticosteroid treated patients could be detected and offered osteoporosis prophylaxis before serious loss of bone density has occurred.

(Ann Rheum Dis 1995; 54: 66-68)

Osteoporosis is an important cause of morbidity in patients who require cortico- steroid therapy. ${ }^{12}$ The adverse effects of corticosteroids on bone mass occur early during the course of treatment ${ }^{2}$ and fractures may occur within weeks of the start of high dose steroid therapy; ${ }^{3}$ once established, corticosteroid induced osteoporosis is difficult to reverse. Several agents including calcitonin, ${ }^{1}$ bisphosphonates, ${ }^{4}$ calcium supplements, ${ }^{5}$ vitamin D analogues, ${ }^{2}$ and hormone replacement therapy (HRT), ${ }^{6}$ have been used to treat established steroid induced osteoporosis. There is, however, no consensus at present as to how and when these agents should be used in the prevention of steroid induced osteoporosis in routine clinical practice. An exception is the case of calcium supplementation, which most authorities agree should be given routinely to steroid treated patients. ${ }^{157}$ There is also evidence to suggest that (in the absence of a contraindication) HRT should be given to postmenopausal women receiving steroids. ${ }^{6} 8$ Although steroid induced osteoporosis is a common disorder, the prevalence of steroid usage and the degree to which coprescription of osteoporosis prophylaxis is given are unclear. In this study, we audited corticosteroid prescriptions in relation to prescription of prophylaxis against osteoporosis in a large teaching hospital in the United Kingdom.

\section{Methods}

The study was based in Aberdeen Royal Infirmary-a teaching hospital comprising 1022 beds serving a population of approximately 0.5 million in the north east of Scotland. Our aim was to find out how commonly corticosteroids were prescribed, to study the indications for treatment and duration of therapy, and to define how many patients were also receiving other drug therapy as prophylaxis against the development of osteoporosis. To identify patients, we utilised the established system of prescription monitoring by pharmacists which is conducted on a daily basis in this hospital and in most acute general hospitals in the United Kingdom, mainland Europe and the USA. For each patient who was receiving systemic corticosteroids (i.e. oral or intravenous), the ward pharmacist completed a standard proforma, asking for relevant details such as age, sex, menopausal status (postmenopausal defined as more than two years since last menstrual period), indication for steroid treatment, steroid dose, duration of therapy (either actual

\author{
Polwarth Building, \\ Aberdeen AB9 2ZD \\ United Kingdom. \\ Accepted for publication \\ 4 August 1994 \\ Correspondence to: \\ Dr Stuart H Ralston, \\ University Department of
}


duration of therapy or, in the case of new prescriptions, the attending physician's estimate of how long steroid therapy would need to be continued). Co-prescription of other drugs (calcium supplements, HRT, vitamin $\mathrm{D}$ analogues, bisphosphonates, calcitonin) known to have effects on osteoporosis were also noted. Each record form was completed during the course of the pharmacist's normal daily duties and took between five and 10 minutes to complete. The $\chi^{2}$ test was used in statistical analysis.

\section{Results}

Corticosteroids were prescribed to 214 patients during the three month study period. The median age of the study group was 68.2 years (range 14-91); 96 subjects (45\%) were males. Of the 118 females, $93(79 \%)$ were postmenopausal, $22(18 \cdot 5 \%)$ premenopausal and for three $(2.5 \%)$ the menopausal status was not recorded.

The most common indications for steroids were chronic chest disease $(n=84 ; 39.2 \%)$, neoplasia $(n=17 ; 7 \cdot 9 \%)$, inflammatory bowel disease $(n=16 ; 7 \cdot 4 \%)$, connective tissue diseases/arthritis $(n=16 ; 7 \cdot 4 \%)$, renal disease/ transplant $(n=7 ; 3.3 \%)$, and skin disease $(\mathrm{n}=4 ; 1.8 \%)$; the remainder $(\mathrm{n}=70,32.7 \%)$ comprised a wide variety of other disorders (details not shown).

Figure 1 shows the intended duration of therapy in relation to the indication for treatment for the 178 patients for whom full data were available (duration of therapy was not known or was unrecorded in 36 cases). For the purpose of analysis, steroid therapy was arbitrarily considered 'short term' in those who had been treated for four months or less and 'long term' in patients treated for more than four months. Using this definition, the majority of patients with chest disease were receiving short term steroid therapy $(n=54 ; 65 \%)$, as were patients with inflammatory bowel disease $(13 / 16 ; 81 \%)$ and cancer $(13 / 17 ; 76 \%)$. In contrast, most patients with connective tissue disease, rheumatoid arthritis, and renal disease were receiving long term steroid therapy

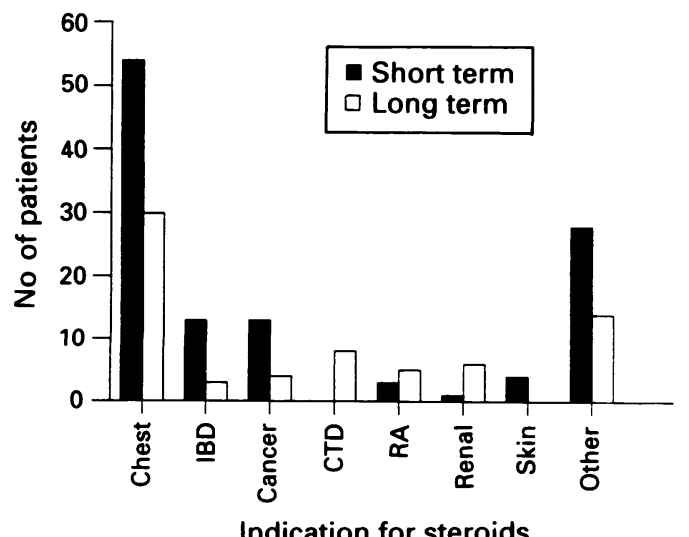

Figure 1 Indications for steroid therapy in relation to duration of therapy. Data are for 178 patients where available. Short term = less than four months; long term $=$ four months or more. IBD = Inflammatory bowel disease; $C T D=$ connective tissue disease; $R A=$ rheumatoid arthritis. $(\mathrm{n}=19 / 27 ; 70 \%)$. This difference (chest disease, inflammatory bowel disease, and cancer $v$ connective tissue disease, arthritis, and renal disease) was highly significant $\left(\chi^{2}=20.8 ; \mathrm{df}=1 ; \mathrm{p}<0.001\right)$.

The dose of steroid (expressed in prednisolone equivalents) varied from $1.6 \mathrm{mg} /$ day to $1000 \mathrm{mg} /$ day (median $32 \mathrm{mg} /$ day). Figure 2 shows the relationship between dose of steroid and expected or actual duration of therapy for 175 patients for whom full data were available. As expected, there was a highly significant inverse relationship between duration of therapy and dose, with the higher doses being prescribed short term and lower doses long term $\left(\chi^{2}=79.9 ; \mathrm{df}=4 ; \mathrm{p}<0.0001\right)$. Importantly, however, 24 of 175 patients $(13.7 \%)$ were receiving high dose steroid therapy ( $>10 \mathrm{mg} /$ day) for more than four months.

Only 12 of the 214 patients $(5 \cdot 6 \%)$ were taking treatment which could be considered to have prophylactic potential to prevent osteoporosis. Of the 93 postmenopausal women studied, six $(6 \cdot 4 \%)$ received prophylaxis and of the 25 patients receiving high dose long term steroid therapy $(>10 \mathrm{mg} /$ day for more than four months), two ( $8 \%$ ) received prophylaxis (difference not significant). Prophylactic therapies given included HRT $(n=5)$, cyclical etidronate $(n=3)$, and vitamin $D$ or calcium alone (two patients).

\section{Discussion}

Our study highlights the fact that systemic steroid treatment is used frequently in clinical practice over a wide range of indications. In our hospital, 214 systemic corticosteroid prescriptions were issued during the study, giving an overall rate of 2.5 new prescriptions every day. In about $50 \%$ of these patients, steroids were given on a long term basis, increasing the potential risk of osteoporosis. ${ }^{1}$ Although routine use of prophylaxis with calcium supplementation and HRT for postmenopausal women have been recommended for such individuals, ${ }^{15-7}$ only $5 \%$ of our study group received any form of prophylaxis against steroid induced osteoporosis. The same was true in high risk patients such as those receiving high dose steroid therapy over a prolonged period of time, and postmenopausal women.

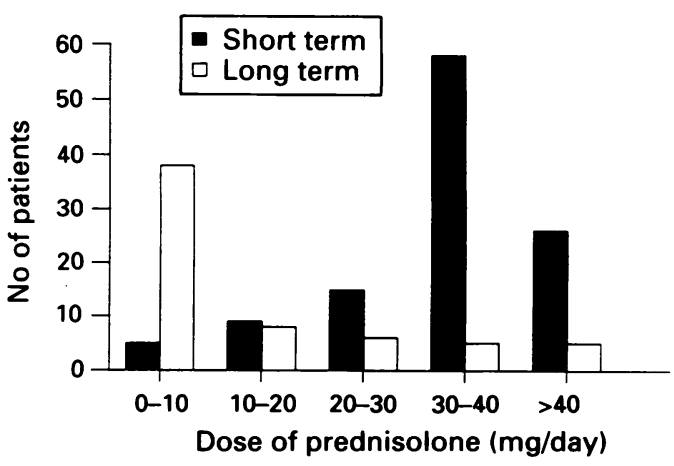

Figure 2 Duration of steroid therapy in relation to dose. Data are for 175 patients where available. Short term $=$ less than four months; long term = four months or more. 
This suggests that even high risk patients who begin taking steroids are not being identified and targeted effectively for prophylaxis. A recent study drew attention to a similar situation in patients with atrial fibrillation, few of whom received anticoagulants as prophylaxis against stroke, despite evidence to suggest that such therapy may be beneficial. ${ }^{10}$

Recent developments highlight the importance of giving prophylactic therapy at an early stage during steroid treatment. ${ }^{2}$ We acknowledge that many of the regimens which have been advocated for the treatment of steroid induced osteoporosis have not been extensively studied in the prevention of such osteoporosis and many are not yet licensed for this indication in the United Kingdom or other countries. Our studies show, however, that two licensed treatments which are available and recommended for use in this situationcalcium supplements ${ }^{5}$ and HRT $^{6}{ }^{11}$-are not being prescribed in routine practice.

The mechanism used in this study to identify patients is widely applicable in routine clinical practice. It is already in place in most hospitals in the United Kingdom, Europe and the USA and provides an effective and extremely cost efficient means by which patients who are receiving corticosteroids can be identified and clinicians alerted to the need for prophylactic therapy. Further studies will be required to evaluate new treatments and to decide whether it is more cost effective to prescribe these therapies to all patients, or to prescribe only to high risk groups, taking into account other factors such as bone mineral density. At present, however, our study highlights the need for wider use of simple therapies for prevention of osteoporosis in patients receiving corticosteroids and provides a mechanism by which clinicians can identify and target these patients for therapeutic intervention.

We thank all the ward pharmacists of Aberdeen Royal Infirmary who contributed to the collection of data and our Consultan who contributed to the collection of data and our Consultant
colleagues who kindly allowed us access to patients under their care.

1 Reid I R, Grey A B Corticosteroid osteoporosis. In Reid D M, ed. Bailliere's Clinical Rheumatology: Osteoporosis. London: Baillière Tindall, 1993; 7: 573-87.

2 Sambrook P, Birmingham J, Kelly P, et al. Prevention of steroid osteoporosis. A comparison of calcium, calcitriol, and calcitonin. N Engl f Med 1993; 328: 1747-52.

3 Bowland E W, Headly N E. Management of rheumatoid arthritis with smaller (maintenance) doses of cortisone acetate. $\mathcal{F} A M A$ 1950; 144: 365-72.

4 Reid I R, King R A, Alexander C J, Ibbertson H K. Prevention of corticosteroid induced osteoporosis with (3-amino-1-hydroxypropylidene)-1,1-bisphosphonate (APD) Lancet 1988; i: 143-6.

5 Reid I R, Grey A B. Calcium supplements in the prevention of steroid induced osteoporosis. Am $\mathcal{F}$ Clin Nutr 1986; 44: 287-90.

6 Lukert B P, Johnston B E, Robinson R G. Estrogen and progesterone replacement therapy reduces glucocorticoid-induced bone loss. $\mathcal{F}$ Bone Miner Res 1992; 7 1063-9.

7 Baylink D J. Glucocorticoid induced osteoporosis. N Engl f Med 1983; 309: 307-8.

8 Meuniere $\mathrm{P}$ J. Is steroid induced osteoporosis preventable? N Engl f Med 1993; 328: 1781-2.

9 Mulder $\mathrm{H}$, Struys A. Intermittent cyclical etidronate in the prevention of corticosteroid induced bone loss. $\mathrm{Br} f$ Rheumatol 1994; 33: 348-50.

10 Bath P M W, Prasad A, Brown M M, MacGregor G A. Survey of use of anticoagulation in patients with atrial fibrillation. BMF 1993; 307: 1045 .

11 British National Formulary, No. 25. London: British Medical Association and the Royal Pharmaceutical Society of Great Britain, 1993; 271-353. 